\title{
Abklärung und Therapie der Varikose - Bilan et traitement de la maladie variqueuse
}

Die Einführung der endovenösen Thermoablation als - in den unkomplizierten Standardsituationen - ebenbürtige Alternative zur klassischen Varizenchirurgie mit Krossektomie und Stripping der Stammvenen hat in den letzten Jahren in der Schweiz in zunehmender Masse zu einer Verunsicherung der Krankenversicherer und Zurückweisungen von Arztrechnungen geführt, selbst wenn diese korrekt waren.

Die Foederatio Medicorum Helveticorum (FMH) hat die ambulante und stationäre Fakturierung medizinscher Leistung dem „WZW-Prinzip“ (Wirksamkeit-Zweckmässigkeit-Wirtschaftlichkeit) unterstellt. Die Schweizerische Gesellschaft für Phlebologie, die Schweizerische Gesellschaft für Angiologie, die Schweizerische Gesellschaft für Gefäßchirurgie sowie die Union der Schweizerischen Gesellschaft für Gefäßkrankheiten stellen sich voll und ganz hinter dieses Grundprinzip. Sie unterstützen in Einklang mit dem Code of Conduct der Foederatio Medicorum Chirurgicorum Helvetica (FMCH) den kürzlich publizierten Verhaltenskodex zu Leitlinienkonformität, Diagnostik und Therapie nach den Regeln der ärztlichen Kunst und zur korrekten Fakturierung erbrachter ärztlicher Leistungen (https://fmch.ch/wp-content/uploads/ 2020/01/Code_of_Behaviour_DE.pdf).

Im April dieses Jahres erschien in der Schweizerischen Ärztezeitung eine Stellungnahme der SGP, SGA, SGG und USGG zum modernen Standard der Abklärung und Therapie der medizinisch relevanten Varikosis sowie deren Abgrenzung von ästhetisch indizierten phlebologischen Leistungen, wie beispielsweise der Behandlung der Besenreiservarikose. Zu letzterem Thema hatte die SGP im Artikel von P. Kern bereits im Jahr 2004 klar Stellung bezogen (Schweizerische Ärztezeitung 2004; 85: 2071-2071).

Die ärztliche Weiterbildung in Phlebologie basiert aktuell in der Schweiz auf 3 interdisziplinären Fähigkeitsausweisen:

1. Fähigkeitsausweis (USGG/SIWF/FMH) für Phlebologie https://siwf.ch/files/pdf19/phlebologie_ version_internet_d.pdf

2. Fähigkeitsausweis SGUM für Duplex der peripheren Gefäße, Submodul periphere Venen

http://www.sgum.ch/sektionen/gefaesse/ dokumente/ausfuehrungsbestimmun gen_gefaesse_2018.pdf

3. Fähigkeitsausweis (USGG/SIWF/FMH) für endovenöse Thermoablation der Stammvenen bei Varikosis https://www.siwf.ch/files/pdf19/ fa_endov_thermoablation_d.pdf

Wichtige ethische Leitlinien zu standesgemäßem ärztlichem Verhalten bilden die Standesordnung der FMH (https://www.fmh.ch/files/ pdf7/standesordnung-fmh.pdf), der Code of Conduct der FMCH (siehe oben) sowie der moderne Ärzte-Eid (https://fmch.ch/ bereiche/ethik/schweizer-eid/).
Ein zentrales Anliegen des vor kurzem in der Schweizerischen Ärztezeitung publizierten Artikels bildet der Krankheitswert der medizinisch relevanten Varikose. Es geht nicht an, dass das Laienpublikum oder die Versicherer sämtliche Formen der Varikose in den Topf der ästhetischen Medizin werfen. Ein großer Anteil der phlebologischen Routine betrifft medizinisch relevante Befunde, welche unbehandelt zu schweren Formen der chronischen venösen Insuffizienz, zum dekompensierten postthrombotischen Syndrom, zum venösen Ulkus, zu Arbeitsunfähigkeit und zu Invalidität führen können.

Andererseits ist es nicht statthaft, ästhetische phlebologische Leistungen zulasten der Krankenversicherer abzurechnen. Die beiden Artikel von Kern (SÄZ 2004; 85: 2070-2071) und von Traber (SÄZ 2020; 101: 632-637) schaffen diesbezüglich eine schriftlich formulierte, klare Orientierung zuhanden (A) der betroffenen Patientinnen und Patienten, Angehörigen und Laien, (B) der Leistungserbringer: Ärzteschaft, Physiotherapie, Bandagistenberufe, Kompressionsmittelhersteller und (C) last not least der Leistungsträger = Krankenversicherer.

Jürg Traber, Daniel Staub, Matthias K. Widmer, Corina Canova, Stefan Küpfer, Philippe Kern, Paolo Cassina, Dominik Heim, Nicolas Ducrey, Rosmarie Holzinger, Christina Jeanneret, Jürg Hafner 